\title{
Gestational Diabetes Mellitus in Primi Gravida of Bangladesh in Different Trimesters
}

\author{
Ifat Ara Begum ${ }^{1}$ \\ ${ }^{1}$ Department of Biochemistry, Dhaka Medical College, Dhaka, Bangladesh \\ Correspondence: Ifat Ara Begum, Asset Avalon, Flat-B/2, House-23, Road-1, Sector-6, Uttara, Dhaka, \\ Bangladesh. Tel: 880-181-931-7616. E-mail: ifat72@yahoo.com
}

Received: February 24, 2014 Accepted: March 23, $2014 \quad$ Online Published: April 2, 2014

doi:10.5539/ijb.v6n3p18 URL: http://dx.doi.org/10.5539/ijb.v6n3p18

\begin{abstract}
Background: Gestational Diabetes Mellitus is defined as any degree of glucose intolerance with onset or first recognition during pregnancy. It is an important dimension of the syndrome of Diabetes Mellitus. Similar to other members of the Asian race, Bangladeshi women are also considered to be at a high risk for developing gestational diabetes.

Materials and Methods: In order to better understand whether this heightened risk attributed to race really exists even in primi gravida of Bangladesh, what is the percentage of the disease among them and whether there is any association of classical risk factors of the disease, a hospital based observational study was performed and the glycemic status of the primi gravida women presenting to Chittagong Medical College Hospital were assessed.

Results: A total of 117 primi gravida women, 39 in each of the three trimesters of pregnancy were selected as study subjects during the study period on the basis of set criteria and a $2 \mathrm{~h}, 75 \mathrm{~g}$ OGTT was performed over them. The mean age of the subjects enrolled was $21.1 \pm 2.29$ years, mean BMI was $21.6 \pm 2.72 \mathrm{~kg} / \mathrm{m}^{2}$, the mean fasting serum glucose values in $\mathrm{mmol} / 1$ were $4.5 \pm 0.63,4.6 \pm 0.62$ and $4.7 \pm 0.56$ in $1 \mathrm{st}, 2 \mathrm{nd}$ and $3 \mathrm{rd}$ trimesters and the $2 \mathrm{~h}$ post $75 \mathrm{~g}$ glucose load serum glucose values in mmol/1 were found $6.1 \pm 1.08,6.6 \pm 1.76$ and $7.0 \pm 1.42$ respectively. Using the cut-off value mentioned in the operational definition of GDM, 13.7\% of the subjects were found to have the disease. Among them, 12.5\% were in first trimester, 31.2\% were in second trimester and 56.3 $\%$ were in third trimester of pregnancy. Maternal obesity/over-weight and a family history of diabetes, not the maternal older age, have shown statistically significant association with GDM. Prevalence of GDM has been noted in clinically non-risk group of subjects also.
\end{abstract}

Conclusion: From the above study, the inference could be drawn that, screening for GDM should be done as early as possible during pregnancy, even at first trimester, irrespective of presence or absence of established risk factors. But larger trials are needed to truly assess the disease burden of gestational diabetes among primi gravida women.

Keywords: Gestational Diabetes, hyperglycemia, trimester of pregnancy, Oral Glucose Tolerance Test

\section{Introduction}

Gestational Diabetes Mellitus is defined as any degree of glucose intolerance with onset or first recognition during pregnancy (The Expert Committee on the Diagnosis and Classification of Diabetes Mellitus, 2003; Buchanan \& Xiang, 2005; American Diabetes Association, 2002; WHO Consultation, 1999; Schmidt et al., 2001). $1 \%$ to $14 \%$ of total pregnancies may be affected by it (American Diabetes Association, 2002). The prevalence of GDM in rural Bangladesh is comparable with any other population with higher prevalence of GDM (Sayeed et al., 2005).

Pregnancy is a complex endocrine-metabolic adaptation process. Placental secretion of hormones, such as estrogen, progesterone, human chorionic somatotropin (hCS) or placental lactogen, prolactin and growth hormone is a major contributor to the insulin resistant state seen in pregnancy (Cianni et al., 2003; Setji et al., 2005) and this insulin resistance, which develops in 2nd trimester, makes pregnancy a diabetogenic condition.

As majority of women with GDM have no symptoms, a screening program is required (Jensen et al., 2003). Risk assessment for GDM should be done at the first prenatal visit (American Diabetes Association, 2002; Murthy et 
al., 2002). The peak effect of maternal hormones responsible for insulin resistance, which is seen in the 26th to the 33rd weeks of gestation, forms the basis for screening in the 24th to the 28th weeks of gestation (Mumtaz et al., 2000). But as diabetes should be diagnosed at the pre-pregnant state and as the patient does not come during this period, glucose screening should ideally be done at the 1st trimester of pregnancy and followed up at the 24 th to the 28th weeks of gestation. But normal glucose tolerance in early pregnancy does not exclude the risk of development of GDM later. Screening earlier in pregnancy detects fewer women with GDM but identifies those at highest risk and allows for earlier intervention. Screening later in pregnancy detects a large number of women with GDM, many of whom are at lower risk but who would be treated for a shorter time (U.S. Preventive Services Task Force, 1996).

The ADA considers women to be at risk for GDM, unless they are younger than 25 years, have normal body weight (BMI $<25 \mathrm{~kg} / \mathrm{m}^{2}$ ), are not a member of high-risk ethnic group, have no first-degree relatives with diabetes and have no personal history of glucose intolerance or poor obstetrical outcome. But screening using only clinical and historic risk factors fails to diagnose the actual percentage of patient with GDM (Mumtaz et al., 2000; Khine, Winklestin, \& Copel, 1999).

In a study of diabetes in pregnancy in Tianjin, China, gravidas with IGT were found to have poor pregnancy outcome, they were found to be at increased risk for premature rupture of membrane, pre-term birth, breech presentation and high birth weight, adjusting for maternal age, pre gravid BMI, hospital levels and other confounding factors (Yang et al., 2002).

There is significant association between increasing glucose intolerance and increased incidence of cesarean delivery, pre-eclampsia and length of maternal hospitalization (Mumtaz et al., 2000).

Gravidas with GDM generally demonstrates higher degrees of post-pregnancy insulin resistance, B-cell dysfunction, higher BMI, central obesity and exaggerated hyperlipidaemia, which suggests that GDM is a transient manifestation of long standing metabolic dysfunction (Carpenter, 2007).

The prevalence of GDM is proportionate to the incidence of type-2 DM in a given population or ethnic group (Setji et al., 2005; U.S. Preventive Services Task Force, 1996; Senanayake et al., 2006). A study including 435 women with a singleton pregnancy done at Oulu University Hospital, Finland between 1984 and 1994, demonstrated occurrence of clinical type- 1 diabetes in $4.6 \%$ and clinical type- 2 diabetes in $5.3 \%$ of women with past history of GDM during their follow-up period in fertile age (Jarvela et al., 2006).

A study carried out for Japanese pregnant women at Kejo University Hospital from June'96 to December'00, demonstrated the occurrence of gestational hypertension more frequently in patients with mild glucose intolerance or GDM, than in those with normal glucose tolerance (Miyakoshi et al., 2004).

In the fetus or neonate, GDM is associated with higher rates of perinatal mortality, macrosomia, birth trauma, hyperbilirubinemia, polycythemia, hypocalcemia, shoulder's dystocia and neonatal hypoglycemia (Mumtaz et al., 2000; Turok, Ratcliffe, \& Baxley, 2003). The children born to mother with GDM may have a higher incidence of obesity, IGT and DM in late adolescence and young adulthood.

Women with GDM need to be managed properly during pregnancy, as treatment of GDM reduces serious perinatal morbidity and may also improve the women's health related quality of life (Crowther et al., 2005). They should be screened for diabetes 6 weeks postpartum, should be re-classified into IFG, IGT, DM or normoglycemic group, and should be followed up with subsequent screening for the development of diabetes or pre-diabetes (esp. in person who had elevated fasting glucose level during pregnancy (Yang et al., 2002)), thus to reduce the risk for complications of diabetes and avoid conception of future pregnancy in the setting of uncontrolled hyperglycemia (Perkins, Dunn, \& Jagasiasm, 2007; Ratner, 2007).

Present study was designed to determine the percentage of primi gravida, identifiable as GDM, by OGTT, in different trimesters of pregnancy, as there is lack of data relevant to it in Bangladesh. Significant association of well-known risk factors with GDM was also planned to be evaluated.

\section{Materials and Methods}

This study was carried out in the department of Biochemistry, CMC with cooperation of Obstetrics \& Gynecology Out-Patient Department, $\mathrm{CMCH}$, during the period of January 2007 to December 2007. A total of 117 primi gravida, 39 in each gestational trimester, who were not known to have previous history of glucose intolerance, were included in the study. Primi gravida with diagnosed pre gestational glucose intolerance, multiple pregnancy, complicated pregnancy (i.e. pregnancy with APH, PET etc.), diagnosed medical diseases (e.g. heart disease) and diseases requiring long term medical treatment that may affect glucose metabolism (e.g. thyroid disorder) were excluded from the study. Detail history was taken and relevant clinical examination was 
done for each subject. When necessary, a relevant clinical finding of index pregnancy was confirmed by investigation. Purpose and procedure of the study were elaborately explained to them. Only those subjects, willing to cooperate, underwent the investigation after giving informed consent in a prescribed form. They were motivated to come next day after overnight fasting condition and were referred for their usual antenatal advice after the procedure.

Data collection procedure included direct history taking, physical examination and laboratory procedure. Data were taken in a pre-designed data collection sheet. Detail history, clinical examination including anthropometry (height, weight and BMI) was recorded. A 2 h, 75 g OGTT was performed on each subject. Venous blood, taken at fasting condition and then $2 \mathrm{~h}$ after $75 \mathrm{~g}$ of oral glucose load, was collected in plain test tube and allowed clotting at room temperature. Serum was obtained by separation of the blood at $3000 \mathrm{rpm} / \mathrm{min}$ within $30 \mathrm{minutes}$ of collection and was used for determination of glucose concentration by Glucose Oxidase Method (Randox Lab. UK). All blood samples were determined within one hour after sampling. Data were managed using the computer software Statistical Package for Social Sciences (SPSS) for Windows, version 12.00. The categorical variables were expressed in terms of percentage. Results were expressed as mean \pm SD unless specified otherwise. The difference in the percentage was tested for significance by Chi-square test. A P value of $<0.05$ was considered statistically significant.

Operational Definitions:

GDM was defined in this study on the basis of fasting and $2 \mathrm{~h}$ serum glucose values of $\geq 5.3 \mathrm{mmol} / \mathrm{l}$ and $\geq 7.8$ $\mathrm{mmol} / \mathrm{l}$ respectively following a $2 \mathrm{~h}, 75 \mathrm{~g}$ Oral Glucose Tolerance Test. If any one or both of the criteria were fulfilled, the diagnosis of GDM was made.

On the basis of family income per month, socio economic status was set, i.e. Poor: Tk. 1,000-5,000, Average: Tk. 5,001-15,000 and Affluent: Above Tk. 15,000.

Presence of any of the established risk factors for GDM like maternal overweight and obesity (BMI of 25 or more), maternal older age (age of 25 years or more) or a family history of diabetes was used to define the clinically risk group. Clinically non-risk group had none of these risk factors.

\section{Results}

The mean age of the total 117 subjects was found to be $21.1 \pm 2.29$ years and mean BMI, $21.6 \pm 2.72 \mathrm{~kg} / \mathrm{m}^{2}$. Majority of subjects were from urban area, of average socio-economic status, were housewives and had the educational status, below SSC level. 25.6\% of the subjects had at least one established risk factor, so considered as clinically risk group. $13.7 \%$ subjects were found to have GDM. $86.3 \%$ subjects had normal glucose tolerance. Of the subjects with GDM, $12.5 \%$ were found in 1 st trimester, $31.2 \%$ were in 2 nd trimester and $56.3 \%$ were in 3rd trimester of pregnancy (Figure 1).

In subjects with normal glucose tolerance (NGT), the mean fasting serum glucose concentration in 1st, 2nd and 3rd trimesters were 4.5, 4.6 and $4.6 \mathrm{mmol} / \mathrm{l}$ respectively and that of $2 \mathrm{~h}$ after $75 \mathrm{~g}$ of glucose load were $6.0,6.1$ and $6.3 \mathrm{mmol} / 1$ respectively (Figure 2). On the other hand, in subjects with gestational diabetes (GDM), the mean fasting serum glucose concentration in 1st, 2nd and 3rd trimesters were 4.2, 5.4 and $4.9 \mathrm{mmol} / \mathrm{l}$ respectively and that of $2 \mathrm{~h}$ after $75 \mathrm{~g}$ of glucose load were 8.3, 10.2 and $9.1 \mathrm{mmol} / \mathrm{l}$ respectively (Figure 3).

A higher prevalence of GDM was detected in clinically risk group of study subjects (Figure 4). A statistically significant association was found not between maternal older age with GDM but between a positive family history of DM as well as maternal overweight and obesity with GDM.

Distribution of GDM Subjects in Different Trimesters of Pregnancy

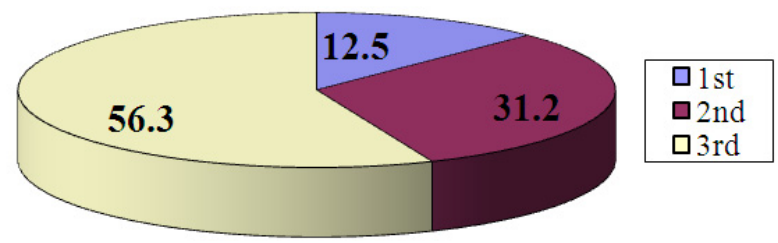

Figure 1. Distribution of GDM subjects in different trimesters of pregnancy 


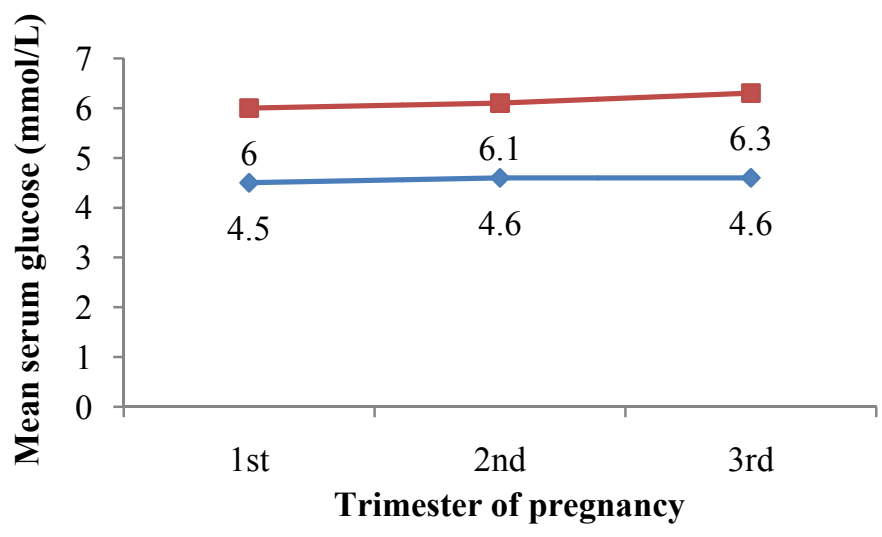

$\prec$ Mean fasting serum glucose

Figure 2. Serum glucose concentrations in subjects with NGT in different trimesters of pregnancy

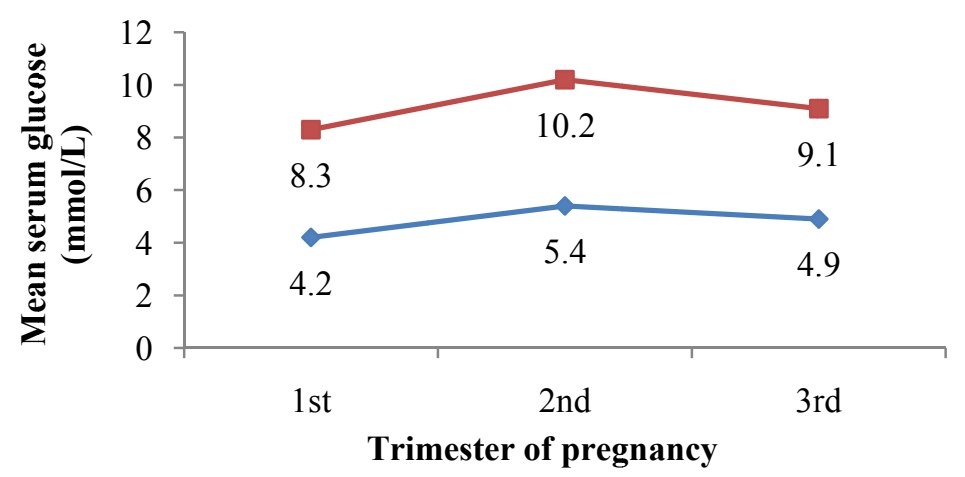

$\leadsto$ Mean fasting serum glucose

Mean serum glucose $2 \mathrm{hr}$ after

Figure 3. Serum glucose concentrations in subjects with GDM in different trimesters of pregnancy

\section{Distribution of GDM Subjects in Clinically Risk Group and Clinically Non-Risk Group}

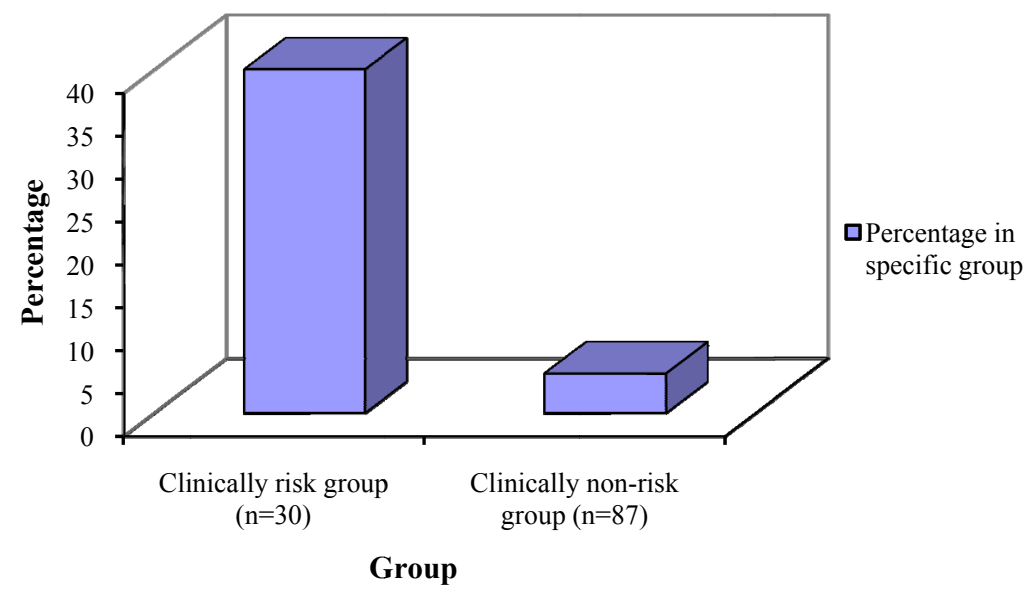

Figure 4. Distribution of GDM subjects in clinically risk group and clinically non-risk group of study subjects 


\section{Discussions}

An overall GDM prevalence of $13.7 \%$ in primi gravida in different gestational trimesters on the basis of a $2 \mathrm{~h}, 75$ g OGTT, as per revealed in the present study, is comparable with any other population with higher prevalence of GDM. The observed difference in GDM prevalence in different trimesters has been supported by different studies also. A study at Chennai revealed a prevalence of $16.3 \%$ at first trimester, $22.4 \%$ at second trimester and $65.3 \%$ at third trimester of pregnancy (Seshiah et al., 2007).

As gestational diabetes was diagnosed in clinically non-risk group as well as in first trimester group of study subjects in this study; the need of screening for GDM is strongly justified in all primi gravidas, even in first trimester, irrespective of presence or absence of clinical risk factors in them. Maternal over weight/obesity and family history of diabetes have shown an association with GDM and found to be statistically significant. The association of maternal older age and GDM has not found to be statistically significant here; but further study is required to come to a conclusion about it.

The present study had some limitations as follows:

1) The small size of sample- the result may not be representative of whole Bangladesh.

2) Subjects were selected from hospital in the city, so they do not represent the overall situation in our country.

3) OGTT was not well accepted by some subjects, esp. in early trimester, when morning sickness sets in. Some subjects started vomiting who were discarded from the test procedure and advised to come later. Many of them dropped out from the study.

4) The initial diagnosis of GDM could not be confirmed by a repeat OGTT on the consecutive day.

\section{Recommendation}

1) Screening for GDM in primi gravida should be done by OGTT as early as possible, even in first trimester of pregnancy.

2) All primi, irrespective of their clinically risk status, should be screened for GDM.

\section{References}

American Diabetes Association. (2002). Gestational Diabetes Mellitus. Diabetes Care, 25(Supplement 1), 94-96. http://dx.doi.org/10.2337/diacare.25.2007.S94

Buchanan, T. A., \& Xiang, A. H. (2005). Gestational diabetes mellitus. J. Clin. Invest., 115, 485-491. http://dx.doi.org/10.1172/JCI24531

Carpenter, N. W. (2007). Gestational Diabetes Pregnancy Hypertension, and Late Vascular Disease. Diabetes Care, 30(Supplement 2), 246-250. http://dx.doi.org/10.2337/dc07-s224

Cianni, G. D., Miccoli, R., Volpe, L., Lencioni, C., \& Prato, S. D. (2003). Intermediate metabolism in normal pregnancy and in gestational diabetes. Diabetes Mtab Res. Rev., 19, 259-270. http://dx.doi.org/10.1002/dmrr.390

Crowther, C. A., Hiller, J. E., Moss, J. R., McPhee, A. J., Jeffries, W. S., \& Robinson, J. S. (2005). Effect of Treatment of Gestational Diabetes Mellitus on Pregnancy Outcomes. The New England Journal of Medicine, 352(24). http://dx.doi.org/10.1056/NEJMoa042973

Jarvela, I. Y., Juutinen, J., Koskela, P., Hartikainen, A. L., Kulmala, P., Knip, M., \& Tapanainen, J. S. (2006). Gestational Diabetes Identifies Women at Risk for Permanent Type 1 and Type 2 Diabetes in Fertile Age: Predictive role of autoantibodies. Diabetes Care, 29(3), 607-612. http://dx.doi.org/10.2337/diacare.29.03.06.dc05-1118

Jensen, D. M., Pedersen, L. M., Nielsen, H. B., Westergaard, J. G., Ovesenp, \& Damm, P. (2003). Screening for gestational diabetes mellitus by a model based on risk indicators: A prospective study. Am. J. Obstet. Gynecol., 189(5), 1383-1388. http://dx.doi.org/10.1067/S0002-9378(03)00601-X

Khine, M. L., Winklestin, A., \& Copel, J. A. (1999). Selective screening for gestational diabetes mellitus in $\begin{array}{lllll}\text { adolescent pregnancies. Obstet Gynecol., 93(5, Part 1), } & \text { 738-742. }\end{array}$ http://dx.doi.org/10.1016/S0029-7844(98)00550-X

Miyakoshi, K., Tanaka, M., \& Matsumoto, T. (2004). Hypertensive disorders in Japanese women with gestational glucose intolerance. Diabetes Research and Clinical Practice, 64, 201-205. http://dx.doi.org/10.1016/j.diabres.2003.11.002 
Mumtaz, M. (2000). Gestational Diabetes Mellitus. Malaysian Journal of Medical Sciences, 7(1), 4-9.

Murthy, E. K., Renar, I. P., \& Metelko, Z. (2002). Diabetes and Pregnancy. Diabetologia Croatica, 31(3).

Perkins, J. M., Dunn, J. P., \& Jagasiasm. (2007). Perspectives in Gestational Diabetes Mellitus: A Review of Screening, Diagnosis and Treatment. Clinical Diabetes, 25(2). http://dx.doi.org/10.2337/diaclin.25.2.57

Ratner, R. E. (2007). Prevention of Type 2 Diabetes in Women with Previous Gestational Diabetes. Diabetes Care, 30(Supplement 2), 242-245. http://dx.doi.org/10.2337/dc07-s223

Sayeed, M. A., Mahtab, H., Khanam, P. A., Begum, R., Banu, A., \& Khan, A. K. A. (2005). Diabetes and hypertension in pregnancy in a rural community of Bangladesh: a population-based study. Diabetic Medicine, 22(9), 1267-1271. http://dx.doi.org/10.1111/j.1464-5491.2005.01600.x

Schmidt, M. I., Duncan, B. B., Reichelt, A. J., Branchtein, L., \& Yamashita, T. (2001). Gestational Diabetes Mellitus Diagnosed With a 2 h 75 g Oral Glucose Tolerance Test and Adverse Pregnancy Outcomes. Diabetes Care, 24(7), 151-1155. http://dx.doi.org/10.2337/diacare.24.7.1151

Senanayake, H., Seneviratne, S., Ariyaratne, H., \& Wijeratne, S. (2006). Screening for gestational diabetes mellitus in Southern Asian women. J. Obstet. Gynaecol. Res., 32(3), 286-291. http://dx.doi.org/10.1111/j.1447-0756.2006.00400.x

Seshiah, V., Balaji, V., Balaji, M. S., Paneerselvam, A., Arthi, T., Thamizharasi, M., \& Datta, M. (2007). Gestational diabetes mellitus manifests in all trimesters of pregnancy. Diabetes Research and Clinical Practice, 77, 482-484. http://dx.doi.org/10.1016/j.diabres.2007.01.001

Setji, T. L., Brown, A. J., \& Peinglos, M. N. (2005). Gestational Diabetes Mellitus. Clinical Diabetes, 23(1). http://dx.doi.org/10.2337/diaclin.23.1.17

The Expert Committee on the Diagnosis and Classification of Diabetes Mellitus. (2003). Report of the Expert Committee on the Diagnosis and Classification of Diabetes Mellitus. Diabetes Care, 26(Supplement 1), 5-20. http://dx.doi.org/10.2337/diacare.26.2007.S5

Turok, D. K., Ratcliffe, S. D., \& Baxley, E. G. (2003). Management of gestational diabetes mellitus. American Family Physicians, 68(9).

U.S. Preventive Services Task Force. (1996). Screening for gestational diabetes. Guide to Clinical Preventive Services (2nd Ed.). Washington, DC: Office of Disease Prevention and Health Promotion.

WHO Consultation. (1999). Definition, Diagnosis and Classification of Diabetes Mellitus and its Complication: report of a WHO Consultation. Part 1: Diagnosis and Classification of Diabetes Mellitus. Geneva, WHO/NCD/NCS/99.2, World Health Organization.

Yang, X., Hsuhage, B., Zhang, C., Zhang, H., Zhang, Y., \& Zhang, C. (2002). Women With Imparted Glucose Tolerance During Pregnancy Have Significantly Poor Pregnancy Outcomes. Diabetic Care, 25, 1619-1624. http://dx.doi.org/10.2337/diacare.25.9.1619

\section{Copyrights}

Copyright for this article is retained by the author(s), with first publication rights granted to the journal.

This is an open-access article distributed under the terms and conditions of the Creative Commons Attribution license (http://creativecommons.org/licenses/by/3.0/). 